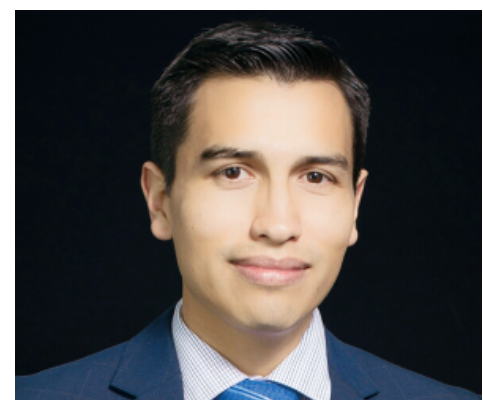

\section{Molares en $C$ tratados con el sistema self adjusting file y sellado de perforaciones con biodentine: Control de dos casos clínicos}

\author{
Dr. Luis Armando Pacheco Ramírez
}

Autor de correspondencia: endo_lap@hotmail.com

\section{C shape molars treated with self-adjusting system file and perforation sealing with biodentine: Control of two clinical cases}

Fecha de recepción: Junio 2018

Fecha de aceptación: Septiembre 2018

\section{Resumen}

La anatomía dentaria tiene diversas variaciones, de acuerdo a la raza, edad, sexo, etc. $Y$ una de ellas son los conductos en forma de $C$, de mayor frecuencia en molares inferiores. La característica anatómica principal de este sistema es la presencia de una red o aleta que conecta los conductos independientes, mientras que el orificio de entrada puede aparecer como una apertura simple en forma de arco de $180^{\circ}{ }^{1-2}$. El presente artículo describe el diagnóstico y manejo clínico de dos molares inferiores con esta característica anatómica, haciendo uso del sistema Self Adjusing File (SAF) durante el tratamiento. Precisamente estos dos casos presentaban perforaciones a nivel endodóntico las cuales se solucionaron usando Biodentine.

Palabras clave: Molar inferior, Conducto en forma de C, Self Adjusting File, Perforación, Biodentine.

\section{Abstract}

Dental anatomy has many variations, according to race, age, sex, etc. One of these are C shape canals, with major frequency in mandibular molars, the main anatomical characteristic of this system is the presence of a network or fin that connects the independent canals, while the entrances orifice can appear as a simple opening in the form of an arc of $180^{\circ}$. The present article describes the diagnosis and clinical management of two lower molars with this anatomical characteristic, using The Self Adjusting File during the treatment. Precisely these two cases presented perforations in the root canal which were solved using Biodentine.

Key words: Lower molar, C shape canal, SelfAdjusting File, operatory microscope.

\footnotetext{
${ }^{1}$ Especialista en Endodoncia, EMI, Bolivia. 


\section{Introducción}

El conocimiento de la morfología radicular es imprescindible para el éxito en la terapia endodóntica. El sistema de conductos en $\mathrm{C}$ es una variación anatómica que está presente de manera más frecuente en segundos molares inferiores, especialmente en poblaciones asiáticas ${ }^{3-4}$. Cooke y Cox describieron por primera vez los conductos en $C$, los cuales presentan mayores desafíos respecto al desbridamiento y obturación ${ }^{3}$. Este tipo de variante anatómica puede ser encontrada en molares superiores e inferiores, así como también en premolares ${ }^{5-6-7-8}$. Estos sistemas de conductos radiculares tienden a tener aletas planas y de gran extensión, lo que puede presentar un desafío aún mayor si hay conexiones en forma de malla entre las aletas.

Si no se toma en cuenta estas variantes anatómicas, podemos causar perforaciones radiculares, para lo cual en el caso que amerite debemos saber repararlas, actualmente contamos con materiales biocerámicos de altísima calidad, entre ellos esta Biodentine compuesto a base de Silicato de calcio el cual tiene fácil manipulación y un tiempo de fraguado corto, entre otras propiedades ${ }^{9}$. Las limas rotatorias de níquel-titanio (NiTi) que se utilizan actualmente son de gran ayuda cuando se trata de conductos curvos simples con secciones transversales redondas. Sin embargo, estos instrumentos son menos efectivos cuando se trata de canales planos y ovales ${ }^{10-11}$. Sin embargo, no se puede visualizar hasta qué punto las aletas bucales y linguales o los istmos no se vieron afectados por el procedimiento endodóntico. Estas partes no tratadas del sistema de conductos radiculares pueden servir como hábitat potencial o paso de bacterias ${ }^{12}$. Los movimientos adicionales de cepillado con limas rotatorias NiTi o instrumentos convencionales de acero inoxidable o NiTi, que comúnmente se cree que abordan la anatomía plana de estos canales, no logran hacer mucha diferencia; todavía dejan más del 50\% de la pared del canal sin cambios, e incluso pueden dejar debris acumulado en los istmos o aletas del conducto 13-14-15-16.

Hace aproximadamente una década apareció un instrumento de características diferentes a los instrumentos rotatorios convencionales, el llamado sistema Self Adjusting File (SAF - ReDent-Nova, Ra'anana, Israel), que es una lima hueca, de paredes delgadas de 1.5 o $2.0 \mathrm{~mm}$ de diámetro compuesto de NiTi de $120 \mu \mathrm{m}$ de espesor. La lima de $1.5 \mathrm{~mm}$ puede comprimirse fácilmente hasta el punto de ser insertado en cualquier canal previamente preparado o negociado con una K-file \#20. La de $2.0 \mathrm{~mm}$ se comprime en un conducto preparado previamente con una K-file \#30.

La lima intenta recuperar sus dimensiones originales, aplicando una presión constante y delicada en las paredes del canal. Cuando se inserta en un conducto radicular, se adapta a la forma del canal, tanto longitudinalmente (como cualquier lima de níquel titanio) y a lo largo de la sección transversal. En un conducto oval o plano alcanza una sección transversal plana u oval, proporcionando una adaptación tridimensional. El diseño hueco permite una irrigación continua durante el procedimiento. Para lo cual existen aparatos dispensadores de irrigante especiales, que se conectan a un tubo de silicona que va directo a la lima. La lima se inserta en un cabezal de contra Angulo especial (RDT3 de ReDentNova) y se programa hasta $5000 \mathrm{rpm}$ a un mínimo torque con una amplitud de $0,4 \mathrm{~mm}$. Se deben hacer movimientos suaves de picoteo de $3 \mathrm{~mm}$ insertando la lima hasta el límite de trabajo durante 4 minutos por conducto con el irrigante que uno prefiera, pero se recomienda Hipoclorito ${ }^{17-18}$.

El objetivo del presente artículo es mostrar brevemente en un par de casos clínicos como esta lima se adapta fácilmente en conductos en $C$ asi mismo cabe mencionar que en ambos casos se utilizó Biodentine para reparar las perforaciones presentes.

\section{CASO CLÍNICO 1}

Paciente de sexo femenino de 23 años de edad llegó a consulta refiriendo dolor agudo en región hemifacial izquierda, incluso hasta zona temporal, a su vez, refería haber iniciado tratamiento de conductos previamente 2 semanas antes y que a partir de la segunda cita fue aumentando el dolor 
progresivamente. A la inspección clínica se observó el diente 3.7 sin obturación temporal y previamente iniciado, con exposición al medio bucal. Dolor a la percusión y palpación. Radiográficamente se observa raíces fusionadas del diente 37 magen 1.

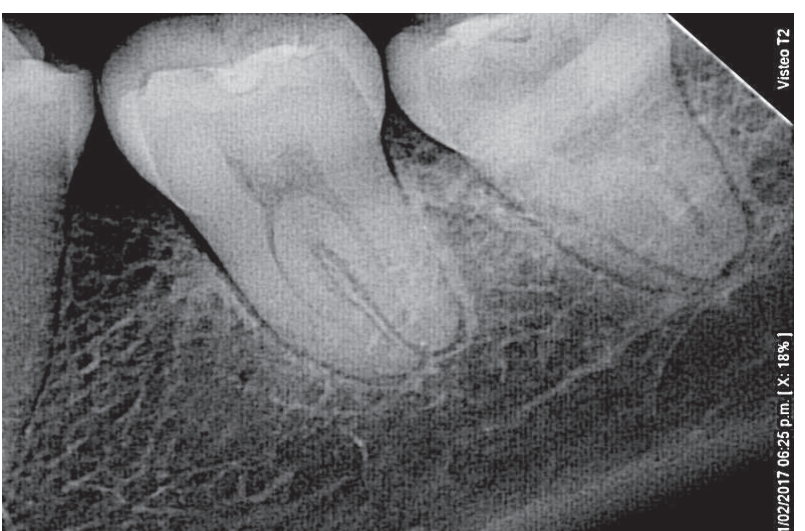

Imagen 1: Radiografía inicial

Se realizó la anestesia usando Septanest (Septodont, Saint Maur de Fossés, France) mediante la técnica troncular del maxilar inferior. Se aisló el diente con dique de goma (NicTone, México) al momento de hacer la exploración del piso cameral no se observó perforación, pero al momento de insertar limas c+10 largo (Dentsply, Maillefer, Ballaigues, Suiza) en conducto mesiovestibular se observó sangrado profuso, al finalizar la primera sesión y después de constatar que ya habían instrumentado mínimamente hasta un calibre de diámetro 30 se usó como medicación intraconductos hidróxido de calcio Metapaste (Meta Biomed Co. Korea) posterior a dejar metapaste solo en conducto distal se tomó una radiografía para observar la desviación y probable perforación ${ }^{\text {Imagen } 2}$ solo para propósito didáctico, después de ello se medicó el resto de los conductos.

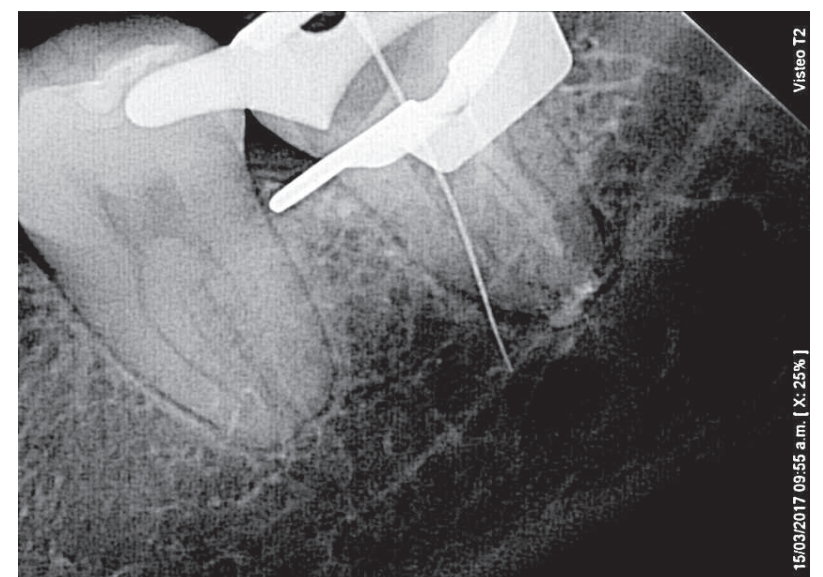

Imagen 2: Metapaste en conductos distales, lima K 20 en conducto mesial.
A partir de la imagen radiográfica se sospechó de la comunicación franca con nervio dentario inferior. Fue realizado un examen complementario de CBCT (Cone Beam Computer Tomography), el cual, a parte de corroborar proximidad con dicho reparo anatómico, mostró la presencia de conducto en C en tercio apical Imagen $3 y 4$.
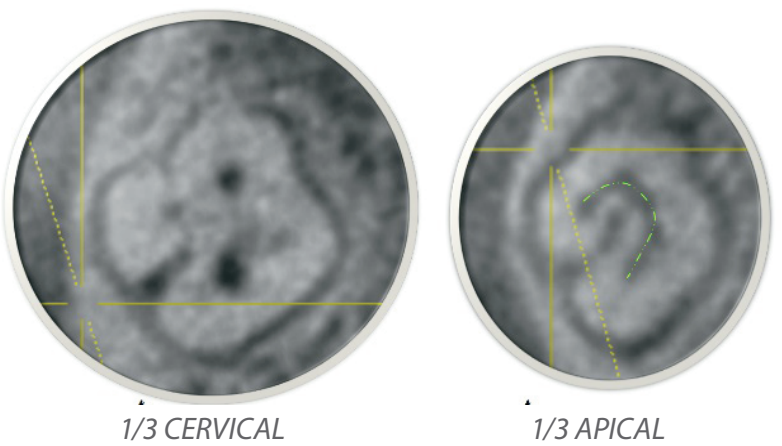

Imagen 3: Corte Disto Vestibular CBCT

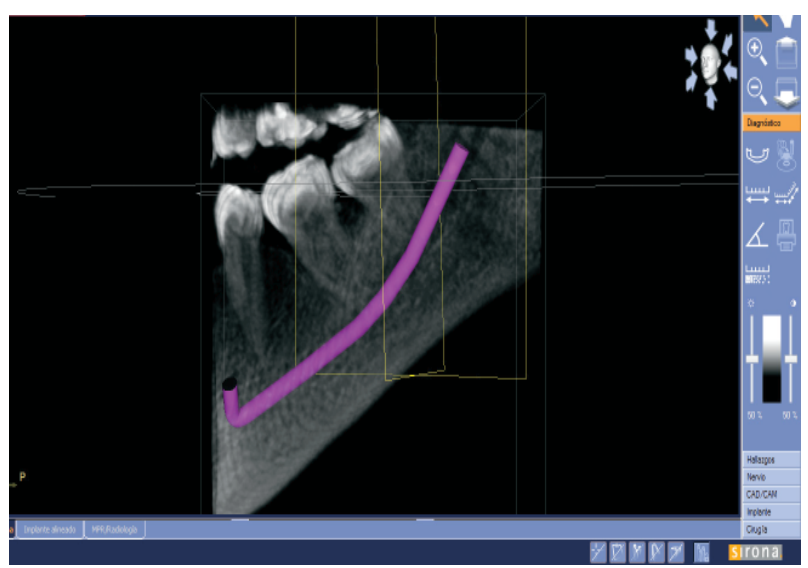

Imagen 4: Corte Oclusal CBCT

En la segunda cita la paciente presentó ligera mejoría. Tomando en cuenta que la perforación se encontraba en tercio medio radicular del conducto mesiovestibular y luego de retomar la luz original del conducto se decidió realizar una reparación de dicha perforación usando Biodentine (Septodont, Saint Maur de Fossés, France) para lo cual y con el objetivo de no obstruir la totalidad de los conductos se usó conos de gutapercha de menor calibre (F1, ProTaper, Dentsply) Imagen 5 y 6 . 


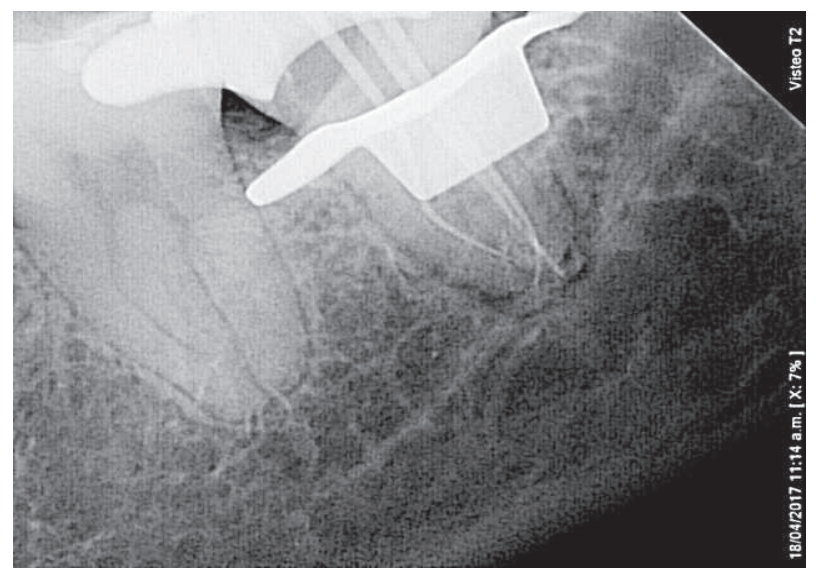

Imagen 5: Conos de gutapercha F1 en conductos

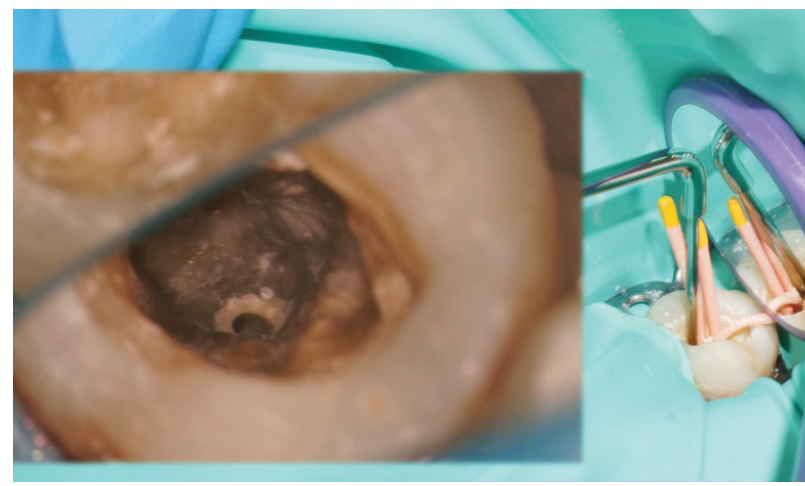

Imagen 6: Conos de gutapercha para evitar la obliteración total del conducto con Biodentine.

El preparo químico mecánico fue realizado con el Sistema SAF (ReDent-Nova, Ra'anana, Israel) Imagen7, usando Hipoclorito de Sodio al 5,25\% colocando el contraangulo a un motor elements (Sybron Endo) a $5000 \mathrm{rpm}$ y $1 \mathrm{~N}$ de torque. Programando el dispensador VATEA (ReDent-Nova, Ra'anana, Israel) a $5 \mathrm{ml}$, accionando la lima durante 4 minutos por conducto, el lavado final fue con EDTA al 17\% y suero fisiológico. Todos los conductos ya habían sido instrumentados previamente hasta llegar a una lima 35/04, por lo tanto, la lima SAF que se usó fue la 2.0.

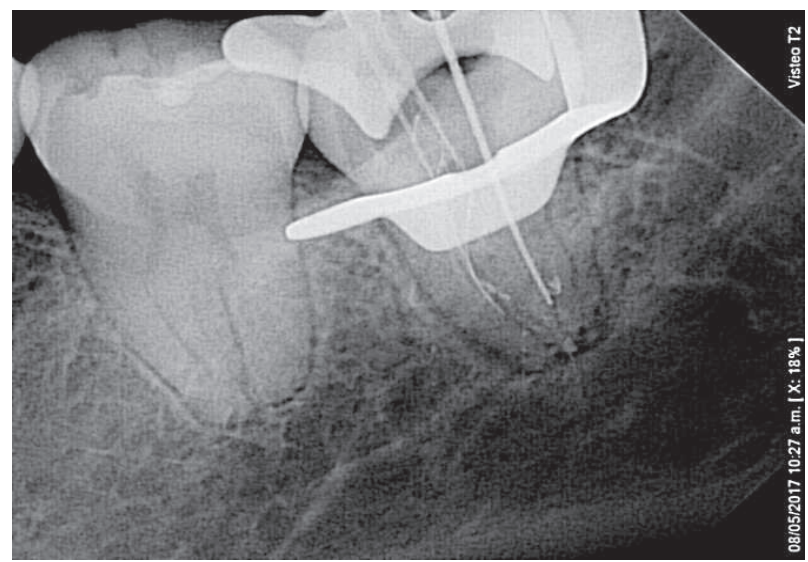

Imagen 7: Lima SAF en conducto mesial
Para proceder con la obturación, se eligió la técnica de ola continua de calor usando el sistema EQ-V (Meta Biomed Co. Korea). Radiográficamente se apreció una obturación adecuada Imagen 8 y 9 .

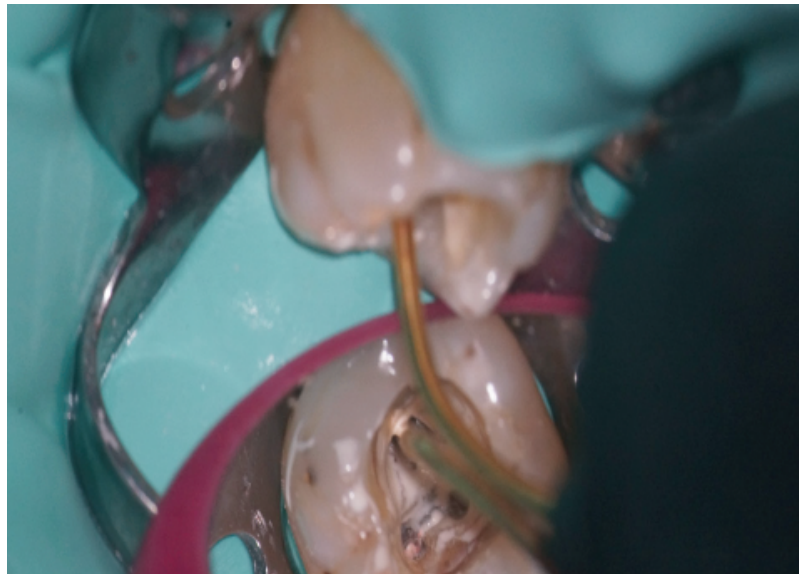

Imagen 8: Obturación con EQ- V (Meta Biomed)

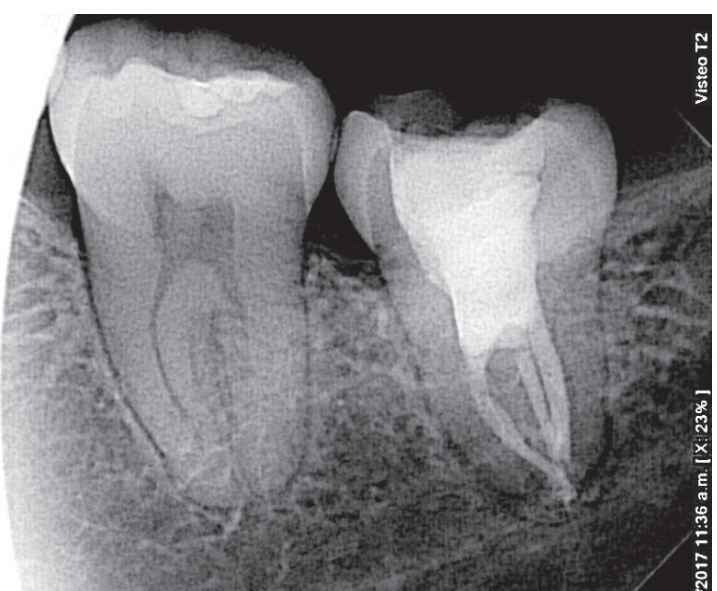

Imagen 9: Radiografía Final

La restauración definitiva fue realizada 1 mes posterior a la finalización del tratamiento de conductos. Los controles fueron realizados en agosto de 2017 y el febrero de 2018 (a un año de iniciado el tratamiento) Imagen $10 \mathrm{y} 11$.

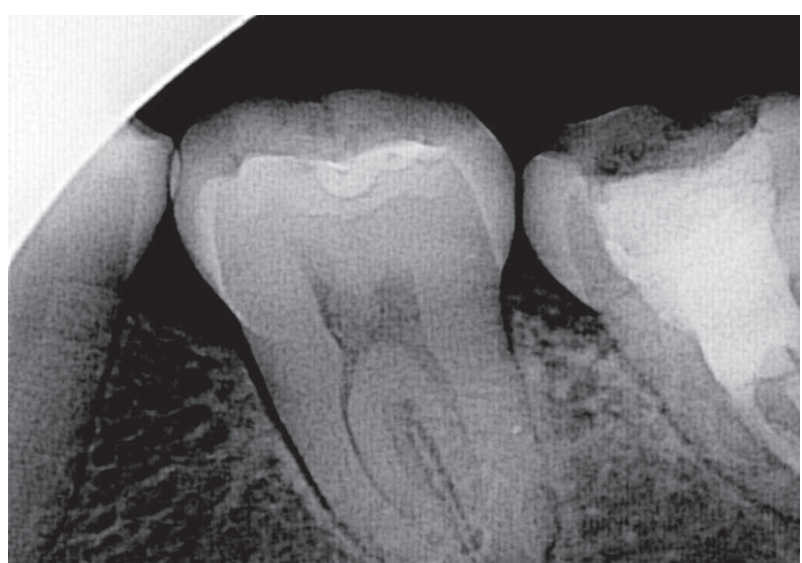

Imagen 10: Control 6 meses 


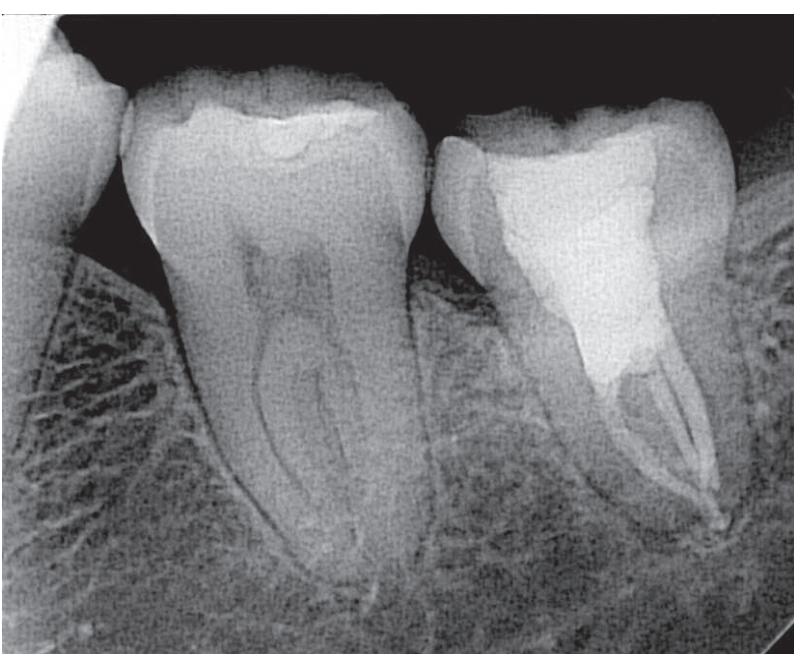

Imagen 11: Control 1 año.

\section{CASO CLÍNICO 2}

Paciente femenino de 22 años fué derivada a consulta, el referidor indicó que existía presencia de perforación en piso de cámara hacia lingual en

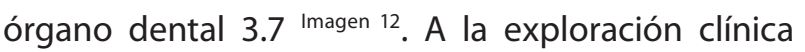
se verificó presencia de dos entradas a conductos mesial y distal.

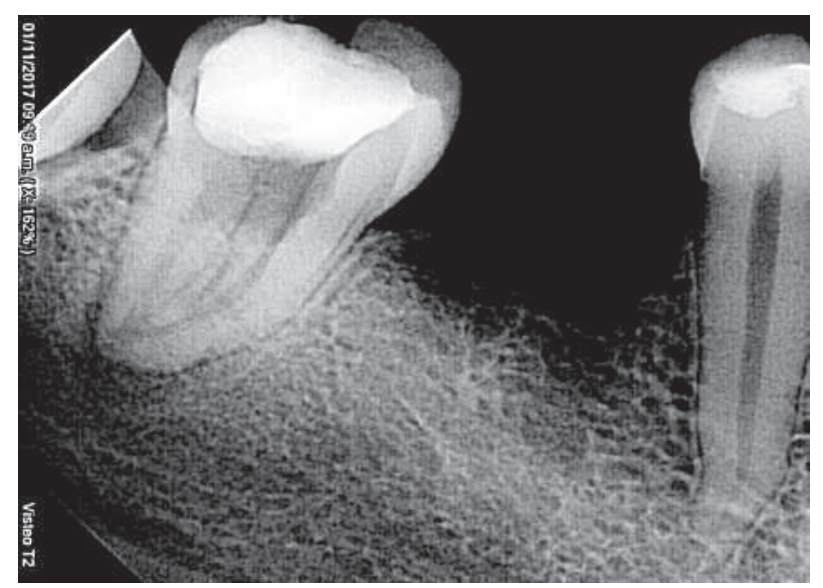

Imágen 12: Radiografía inicial.

Después de colocar anestesia y realizar el aislamiento absoluto se decidió limpiar toda la cámara para proceder a sellar la perforación con Biodentine (Septodont, Saint Maur de Fossés, France) Imágenes 13 a,b.

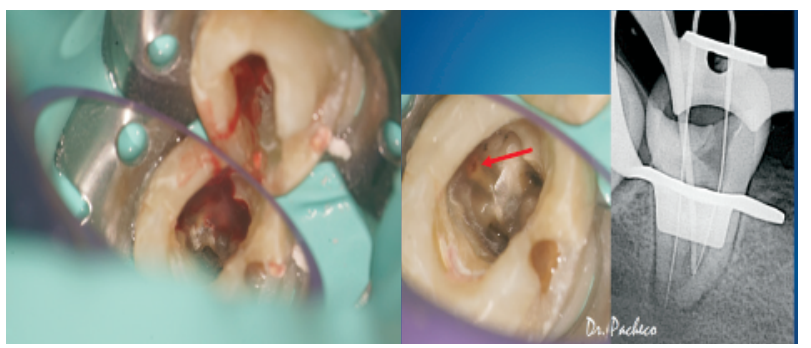

Imagen 13a: Localización y reparación de la perforación con Biodentine.

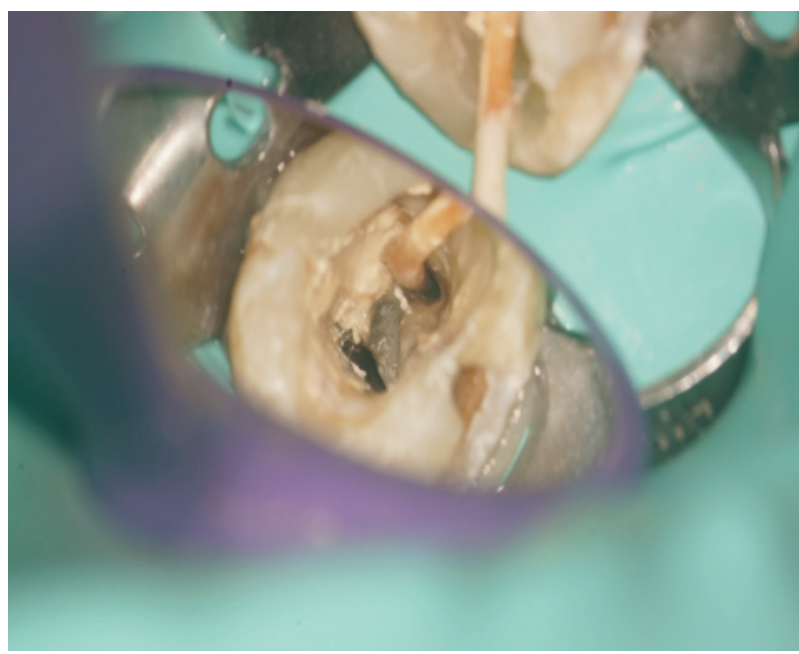

Imagen 13b: Localización y reparación de la perforación con Biodentine.

No se dejó ningún tipo de medicación intraconducto, y en la segunda sesión se instrumentó previamente con limas Hy-Flex (Coltene Whaledent, Cuyahoga Falls, OH, USA) hasta calibre 50/04 en Conducto Distal y 35/04 en Conducto Mesial, constatando que en tercio apical ambos conductos se unían. Posteriormente se utilizó el Sistema SAF (ReDentNova, Ra'anana, Israel|lmagen 14 bajo el siguiente protocolo: SAF 2.0 en cabezal RD3T a 5000 rpm, con hipoclorito de sodio al 5,25\% durante 4 minutos por conducto. Al finalizar se usó EDTA al 17\% con la técnica de irrigación ultrasónica pasiva con Irrisonic (Helse, Brasil) durante 20 segundos, para luego realizar el ultimo lavado con Suero Fisiológico.

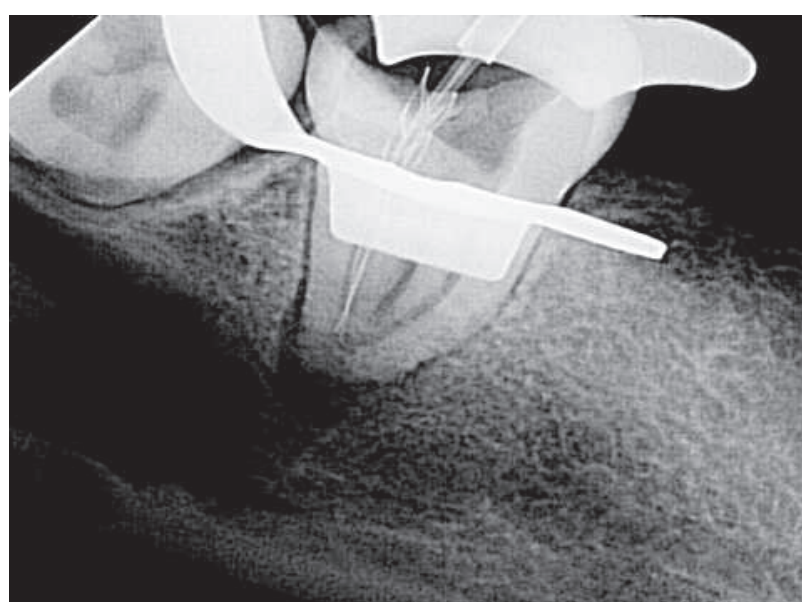

Imagen 14: Lima SAF en conducto distal.

La obturación se la hizo con técnica de ola continua de calor usando el equipo EQ-V (Meta Biomed Co. Korea) $)^{\text {Imagen } 15 .}$ 


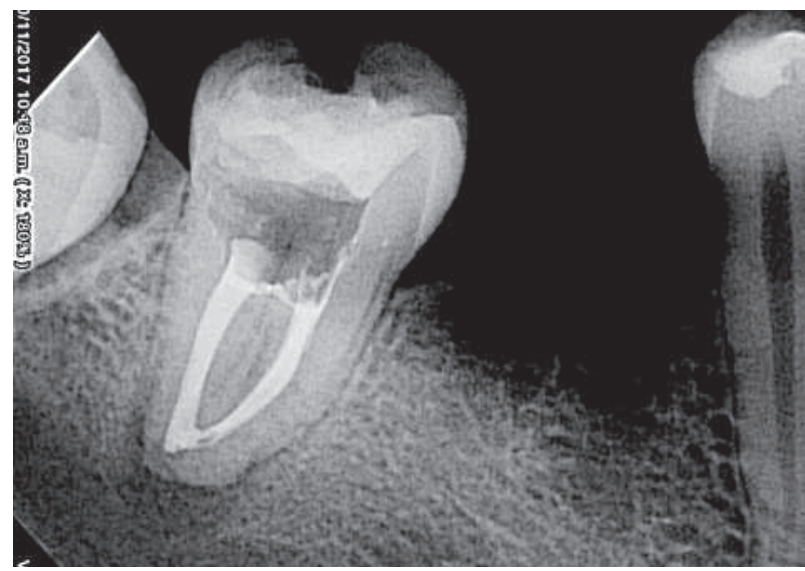

Imagen 15: Radiografía final.

La paciente retornó a su primer control en marzo de 2018 (4 meses de haber finalizado el tratamiento de conductos), sin una restauración definitiva, por lo que se le sugirió que tome contacto con el referidor para culminar el tratamiento.

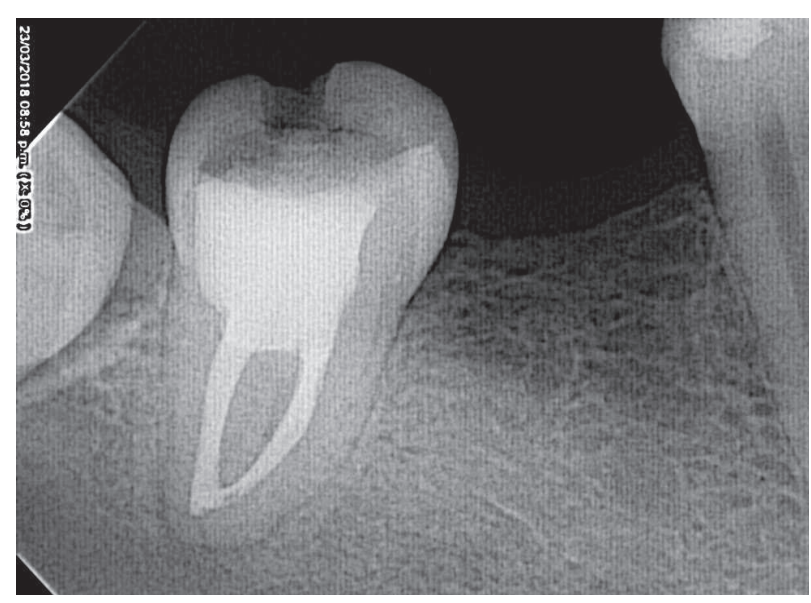

Imagen 16: Control a 4 meses.

\section{Discusión}

La forma y numero de raíces están definidas en las fases embriológicas. Durante la formación radicular la vaina horizontal de Hertwig mantiene la forma de un collar en dientes unirradiculares, por otro lado, si 2 o 3 epitelios crecen hacia el centro del collar, en cierto lugar esas extensiones se encontrarán y fusionarán dejando aberturas para raíces independientes ${ }^{19}$. en un conducto en C estas extensiones no se desarrollan, o su desarrollo fue inadecuado dejando como en estos casos una raíz sin división. La configuración anatómica de este tipo de conductos obliga a usar instrumentos especiales ${ }^{16}$. Especial atención se debe dar a estos casos para evitar perforaciones. El primer problema referente a la perforación fue resuelto gracias a la ayuda del uso de magnificación con Microscopio Clínico (Seiler, Estados Unidos). Y a un nuevo material biocerámico el cual garantiza un buen resultado si se lo maneja adecuadamente.

El uso de un sistema que instrumente e irrigue al mismo tiempo y además de eso se adapte a la anatomía radicular, nos simplifica el desarrollo del tratamiento de conductos radiculares que tienen variaciones anatómicas como ser los conductos en $C$.

\section{Bibliografía}

1. Fan B, Cheung G, Fan M, Gutmann J, Bian Z. C-shaped canal system in mandibular second molars: part I-anatomical features. J Endod. 2004; 30:(899-903.).

2. Gulabivala $\mathrm{K}$, Opasanon A, Ng YL, Alavi A. Root and canal morphology of Thai mandibular molars. Int Endod J. 2002; 35(56-62.)

3. Cooke H, Cox F. C-shaped canal configurations in mandibular molars. J Am Dent Assoc. 1979; 99(836)

4. Lopreite G, Basilaki J. Endodoncia: Criterios técnicos y terapéuticos Ciudad Autonoma de Buenos Aires: Grupo Guia; 2016.

5. De Moor R. C-shaped root canal configuration in maxillary first molars. Int Endod J. 2002; 35(200-8.).

6. Lu T, Yang S, Pai S. Complicated root canal morphology of mandibular first premolar in a Chinese population using the cross section method. J Endod. 2006; 32(932-6)

7. Cleghorn B, Christie W, Dong C. Root and root canal morphology of the human permanent maxillary first molar: a literature review. J Endod. 2006; 32(813-21.)

8. Chai W, Thong Y. Cross-sectional morphology and minimum canal wall widths in C-shaped roots of mandibular molars. J Endod. 2004; 30(509-12.): p. 18:409-18

9. Grech L, Mallia B, Camilleri J. Investigation of the physical properties of tricalcium silicate cement-based root-end filling materials. Dent Mater. 2013; 29(20-8)

10. ElAyouti A, Chu AL, Kimionis I, Klein C, Weiger R, Lost C. Efficacy of rotary instruments with greater taper in preparing oval root canals. Int Endod J. 2008; 41

11. De-Deus G, Miranda-Souza E, Barino B. The Self-Adjusting File optimizes debridement quality in oval-shaped root canals. J Endod. 2011; 37:701-5

12. Siqueira J, Alves F, Almeida B, Machado de Oliveira J, IN R. Ability of chemomechanical preparation with either rotary instruments or Self-adjusting File to disinfect oval-shaped root canals. J Endod. 2010; 36:1860-5

13. Wu MK, Van der Sluis L, Wesselink P. The capability of two hand instrumentation techniques to remove the inner layer of dentine in oval canals. Int Endod J. 2003; 36:218-24.

14. Yin X, Shun-pan CG, Zhang C, Murakami MY, Kimura Y, Matsumoto K. Micro-computed tomographic comparison of nickel-titanium rotary versus traditional instruments in C-shaped root canal system. J Endod. 2010; 36:708-12.

15. Paque F, Laib A, Gautschi H, Zehnder M. Hard-tissue debris accumulation analysis by high-resolution computed tomography scans. J Endod. 2009; 35(1044-7).

16. Solomonov M, Paque F, Fan B, Eilat Y, Berman LH. The Challenge of C-shaped Canal Systems: A Comparative Study of the SelfAdjusting File and ProTaper. J Endod. 2012; 38(2)

17. Flavio RF, Alves B, Almeida M, Neves MAS, Rocas IN, Siqueira JF. Time-dependent Antibacterial Effects of the Self-Adjusting File Used with Two Sodium Hypochlorite Concentrations. J Endod. 2011; 37(10)

18. Metzger Z, Teperovich E, Zary R, Cohen R, Hof R. The Selfadjusting File (SAF). Part 1: Respecting the Root Canal Anatomy-A New Concept of Endodontic Files and Its Implementation. J Endod. 2010; 36(4)

19. Castelucci. Embriology Florence: Tridente; 2004. x 